\title{
PhiMiSci
}

Philosophy and the Mind Sciences

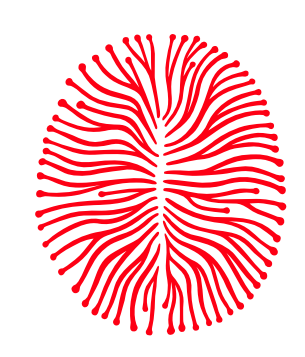

\section{Generality and content-specificity in the study of the neural correlates of perceptual consciousness}

\author{
Tomáš Marvan ${ }^{\mathrm{a}}$ (D) (marvan@flu.cas.cz) \\ Michal Polák ${ }^{\mathrm{b}}$ (D) (miha@kfi.zcu.cz)
}

\begin{abstract}
The present paper was written as a contribution to ongoing methodological debates within the NCC project. We focus on the neural correlates of conscious perceptual episodes. Our claim is that the NCC notion, as applied to conscious perceptual episodes, needs to be reconceptualized. It mixes together the processing related to the perceived contents and the neural substrate of consciousness proper, i.e. mechanisms making the perceptual contents conscious. We thus propose that the perceptual NCC be divided into two constitutive subnotions. The main theoretical idea that emerges as a consequence of this reconceptualization is that the neural correlate of a perceptual episode is formed in the neural interaction between content-processing and consciousness-conferring mechanisms. The paper elaborates this distinction, marshals some initial arguments in its favour, and tests it against some of the most debated theories of consciousness.
\end{abstract}

\section{Keywords}

Content-specific NCC $\cdot$ General neural correlate of consciousness $\cdot$ Neural correlates of consciousness $\cdot$ Perceptual experience $\cdot$ Theories of perceptual consciousness

This article is part of a special issue on "The Neural Correlates of Consciousness", edited by Sascha Benjamin Fink.

\section{Introduction}

The last two decades have brought an avalanche of empirical studies of the neural correlates of consciousness (NCCs). Important new findings are reported almost on a daily basis (for good overviews of recent developments see Koch et al., 2016;

${ }^{a}$ Institute of Philosophy of the Czech Academy of Sciences, Prague, Czech Republic

${ }^{\mathrm{b}}$ Department of Philosophy, University of West Bohemia, Pilsen, Czech Republic 
Förster et al., 2020; Michel \& Morales, 2020; Tsuchiya et al., 2015). Parallel to this burgeoning research programme runs a string of methodological and conceptual studies that are informed by this programme and strive to make its central notions precise and its methods more fruitful. To name just the most influential of them, Chalmers (2000) laid down the canonical definition of an NCC as the minimal neural system the activation of which is sufficient for conscious experience, and addressed a number of important methodological issues of the NCC studies. Miller (2015) highlighted the distinction between correlation and constitution in NCC studies and pointed out the limitations of the framework of minimally sufficient NCCs. Hohwy (2009) emphasized the differences between content- and level-based approaches to NCC research. Bayne et al. (2016) found the traditional unidimensional level-based approaches to consciousness untenable and sketched a multidimensional account of global states of consciousness. Aru, Bachmann, et al. (2012) urged the need to distinguish the prerequisites and consequences of conscious perception from the NCC proper. Finally, Haynes (2009) and Sandberg et al. (2014) argued for investigating the NCCs with multivariate decoding of neural activity patterns. This method, they claim, may establish a tight link between types of conscious experiences and neural processing in distributed brain locations.

The present paper was written as a contribution to this ongoing methodological debate within the NCC project. The notion of NCC is equally applied in research on levels of consciousness (more precisely called "global states of consciousness") and in the studies of contentful conscious mental states (Hohwy, 2009). In the research on levels of consciousness, attempts are made to identify the neural underpinnings of various levels of consciousness such as being alert, dreaming, or being in a minimal conscious state (but see McKilliam (2020), this volume). The other main usage of the NCC concept concerns neural processes correlating with conscious mental states, especially with conscious perceptual states such as seeing a bright sunset. Our focus in this paper is entirely on the neural correlates in this latter sense. We are interested in the neural correlates of conscious perceptual episodes that come and go when the level of consciousness remains more or less constant.

Our claim is that the NCC notion, as applied to conscious perceptual states, needs to be reconceptualized. It mixes together the processing related to the perceived contents and the neural substrate of consciousness proper, i.e. mechanisms making the perceptual contents conscious. We thus propose, in section 2 of the paper, that the perceptual NCC be divided into two constitutive subnotions. The first subnotion covers the content-specific side of the perceptual NCC. This subnotion harbors some of the most vexing puzzles of consciousness science concerning the nature and material realization of consciously perceived contents. The central topic of this paper, though, is the second subnotion, that of the neural process or processes making the perceptual contents, clustered in a perceptual state, conscious.

The division of the perceptual NCC into the two subnotions is not a priori but inspired and informed by perceptual NCC research. That being said, not ev- 
ery theorist of consciousness will endorse the reconceptualization we propose. In section 3 we selectively review the theories of consciousness that we believe are supporting the division as well as those that do not sit easily with it. Section 4 puts forward two central arguments for endorsing the division. The section further motivates the idea that from the neural point of view, an episode of conscious perception arises in the neural interaction between the content-processing and consciousness-conferring mechanisms. The concluding section 5 sketches some advantages of the proposed reconceptualization.

\section{Distilling the general neural correlate of percep- tual consciousness from the neural correlates of perceptual contents}

Applied to episodes of conscious perception, the concept of NCC typically concerns neural processes systematically correlating with a particular type of perceptual experience. That is to say, the perceptual NCC is typically a content-specific correlate. We may speak of the NCC of seeing a face or the NCC of the smell of a rose.

The centrality of this content-specific NCC concept can be illustrated by numerous examples. For instance, Crick (1994, p. 9) speaks about the neural correlate of seeing red. The same emphasis on content-specificity is present in the following formulations: "a neural correlate of consciousness is a specific pattern of brain activity that correlates with particular conscious experiences" (Rees et al., 2002, p. 261); "the minimal set of neuronal events and mechanisms jointly sufficient for a specific conscious percept" (Koch, 2004, p. 16); "content-specific NCC are the neural mechanisms specifying particular phenomenal contents within consciousness, such as colors, faces, places, or thoughts" (Boly et al., 2017, p. 9604; see also Koch et al., 2016, p. 308; Aru, Bachmann, et al., 2012, p. 738). Even one of Chalmers' two famous definitions of NCC, commonly accepted in the field as the definitions of NCC, targets content-specific mechanisms expressing the particular types of conscious percepts: "an NCC (for content) is a minimal neural representational system $\mathrm{N}$ such that representation of a content in $\mathrm{N}$ is sufficient, under conditions C, for representation of that content in consciousness" (Chalmers, 2000, p. 31).

Because the notion of NCC is also used by researchers working on levels of consciousness, it would be desirable to have a specific label for the content-specific perceptual NCC. Neural Correlate of conscious (perceptual) content ("NCcc" for short) suggests itself. The NCccs for particular types of conscious perceptual states differ in their neural details. These differences are reflected in the experienced differences between the percepts. There is an indefinite variety of NCccs, corresponding to the variety of perceptual contents we may consciously experience. In all this diversity, though, the search for the neural correlates of consciousness strives to 
identify a common thread. The pivotal question of consciousness studies is what makes any particular perceptual content conscious.

We believe that to address the pivotal question is to take seriously the possibility that the NCcc is a composite. At its core are the mechanisms preparing the contents we experience. Let us call these content mechanisms Neural Correlates of (perceptual) content ("NCc" for short). The NCc is the part of the NCcc that is entirely responsible for the differences between experienced contents - for example, between the conscious seeing of green and the conscious seeing of blue. ${ }^{1}$ The second part of the NCcc is the topic of this paper. It is the neural mechanism making the perceptual contents conscious. In contrast to the ever-varying processes subsumed under the NCc label, this mechanism is shared across different perceptual NCccs. Whenever this mechanism is activated, and the content-specific processing is in place, conscious perceptual states are present as well. Neural activity correlating with a conscious perception of an apple includes both the activation of content mechanisms presenting the visual features of an apple (NCc) and the modality nonspecific process(es) making the bundle of content features of the apple conscious. Similarly for all other particular conscious experiences across all sensory modalities. We may as well call such common mechanism the mechanism of perceptual consciousness itself.

Let us label this mechanism the general Neural Correlate of (perceptual) Consciousness ("gNCC" for short). This content-nonspecific, domain-general neural process is no less important than the content-preparing mechanisms. In fact, the search for this universal neural mechanism is arguably the ultimate goal of perceptual NCC research. ${ }^{2}$ It is plausible that the science of consciousness will only isolate the gNCC by first amassing a great deal of information about the contentspecific NCccs - both in a single sensory modality as well as across modalities. But mere gathering of more and more content-specific data won't do. The next step, we submit, is to isolate the gNCC. This additional step, though, is rarely made by consciousness theorists. On the contrary, as the definitions of the neural correlates of perceptual consciousness cited above demonstrate, the NCcc is usually

\footnotetext{
${ }^{1}$ In this respect, NCc is similar to Block's (2005, p. 47) notion of core phenomenal NCC, which in turn was inspired by Chalmers' core NCC (2000, p. 26). Core NCC contains the presumed core processes correlating with experiences. Core NCC is a part of the total NCC, the overall brain activity sufficient for any conscious perceptual episode. As the total NCC contains more neural activity than is directly relevant for a particular perceptual episode (Bayne \& Hohwy, 2013, p. 25; Chalmers, 2000, p. 26), NCC research is predominantly focused on the core NCC. The notion of the core NCC emphasizes the qualitative differences between manifestations of such core processes in various conscious states. This is clear not just in Block (2005) and Chalmers (2000), but also in other authors writing about the core NCC, such as Keaton (2015).

${ }^{2}$ For reasons of space, we restricted our attention here to perceptual contents and do not make a parallel case for other types of experiential contents. However, we suppose that the idea of a gNCC could be extended to other types of phenomenal states, such as emotions, thoughts, metacognition, dreams, working-memory contents etc. All conscious experiences, whether perceptual, cognitive, or affective, could share the same type of the gNCC mechanism (implemented in different parts of the brain).
} 
pictured as an undifferentiated whole in which content mechanisms are fused with consciousness conferring mechanism.

The gNCC notion will hopefully become more perspicuous when compared with two somewhat related notions. The first is what Koch et al. (Koch et al., 2016) call full NCC. The full NCC is located in the "posterior Hot Zone" comprising parietal, occipital and temporal areas. It is "the neural substrate supporting experience in general, irrespective of its specific content” (Koch et al., 2016, p. 308). The term appears to have the same meaning as gNCC. However, on closer look, the two concepts are quite different. The full NCC is defined as a "union of the sets of content-specific NCC for all possible contents of experience" (ibid.; see also the same claim in Boly et al. (2017), esp. in Fig. 1 on p. 9604). The full NCC is thus a union of all possible content-specific neural patterns, all possible NCccs. The gNCC is not a union at all. Not being involved in the production of perceptual contents, the gNCC does not implement representational aspects. Further, even if the content mechanisms were all located in the posterior Hot Zone, the gNCC might extend into other regions.

The second notion, Fink's (2016, p. 4) "neural correlate of general consciousness", comes closer to what we have in mind. Consider the following passage:

[W]e may talk of broader and broader types, e.g., red experiences, color experiences, visual experiences, sensory experiences, experience while being awake, and so on. The most general correlate we may be interested in is what distinguishes all conscious mental activity from un- or preconscious mental activity. (Fink, 2016, p. 4)

We agree that the general correlate of consciousness is what distinguishes conscious from unconscious mental activity. The difference is that Fink's idea of the neural correlate of general consciousness seems broader than the gNCC. It seems to involve the neural correlates of global states of consciousness, the processes differentiating between being conscious or unconscious in the global sense. In contrast, the gNCC is limited to episodes of conscious perception. The mechanisms of global states of consciousness are presupposed by the gNCC, but do not form part of it.

In the following, we will further explore the idea that the NCcc, the minimal neural activation sufficient for a particular type of experience (Chalmers, 2000, p. 31; Koch, 2004, p. 16), is a composite of the NCc and gNCC. More precisely, since the spatial metaphor might be misleading, the idea is that NCccs are formed in the neural interactions between the content- and consciousness-mechanisms. We will continue to focus predominantly on the gNCC. So far we have been speaking only about an abstract notion of gNCC. In the next section 3, we will introduce some concrete proposals about how to construe the gNCC. 


\begin{tabular}{|c|c|c|c|}
\hline Abbreviation & Type of the neural correlate & Description & Notes \\
\hline $\mathrm{NCC}$ & $\begin{array}{l}\text { Neural correlate of } \\
\text { consciousness }\end{array}$ & $\begin{array}{l}\text { Comes in two types: level-based } \\
\text { NCC and content-based NCC. } \\
\text { Level-based NCC is the neural } \\
\text { underpinning of levels of } \\
\text { consciousness such as being } \\
\text { awake, being in a coma, or } \\
\text { being anaesthetised. } \\
\text { The content-based NCC is the } \\
\text { minimal neural system whose } \\
\text { activation is sufficient for a } \\
\text { particular type of conscious } \\
\text { experience (Chalmers, 2000). }\end{array}$ & $\begin{array}{l}\text { The content-based NCC as } \\
\text { defined by Chalmers (2000) has } \\
\text { the same extension as the NCcc } \\
\text { below. }\end{array}$ \\
\hline Core NCC & $\begin{array}{l}\text { Core neural correlate of } \\
\text { consciousness }\end{array}$ & $\begin{array}{l}\text { Core neural processes } \\
\text { correlating with experiences. }\end{array}$ & $\begin{array}{l}\text { Term introduced in Chalmers } \\
\text { (2000) and further applied in } \\
\text { Block (2005) and Keaton } \\
\text { (2015). Core NCC equals } \\
\text { content-based NCC. }\end{array}$ \\
\hline $\mathrm{NCcc}$ & $\begin{array}{l}\text { Neural correlate of conscious } \\
\text { (perceptual) contents }\end{array}$ & $\begin{array}{l}\text { Neural correlate of a type of a } \\
\text { conscious perceptual state (such } \\
\text { as hearing an airplane in the } \\
\text { distance) }\end{array}$ & $\begin{array}{l}\text { A dynamic combination of } \mathrm{NCc} \\
\text { and gNCC }\end{array}$ \\
\hline $\mathrm{NCc}$ & $\begin{array}{l}\text { Neural correlate of (perceptual) } \\
\text { content }\end{array}$ & $\begin{array}{l}\text { Neural mechanism/pattern } \\
\text { creating the content of a } \\
\text { perceptual state. }\end{array}$ & $\begin{array}{l}\text { The NCc is that part of the } \\
\text { neural correlate of a conscious } \\
\text { perceptual state that } \\
\text { distinguishes one perceptual } \\
\text { content from another, e.g., the } \\
\text { seeing of blue from the seeing } \\
\text { of green. }\end{array}$ \\
\hline gNCC & $\begin{array}{l}\text { General neural correlate of } \\
\text { (perceptual) consciousness }\end{array}$ & $\begin{array}{l}\text { Neural mechanism conferring } \\
\text { consciousness on perceptual } \\
\text { contents. }\end{array}$ & $\begin{array}{l}\text { Free from all content-specific } \\
\text { (representational) aspects. A } \\
\text { somewhat related concept of the } \\
\text { neural correlate of general } \\
\text { consciousness can be found in } \\
\text { Fink (2016). }\end{array}$ \\
\hline Full NCC & $\begin{array}{l}\text { Full neural correlate of } \\
\text { consciousness }\end{array}$ & $\begin{array}{l}\text { Union of the sets of content- } \\
\text { specific NCC for all possible } \\
\text { contents of experience; situated } \\
\text { in the posterior Hot Zone. }\end{array}$ & $\begin{array}{l}\text { Term introduced in Koch et al. } \\
\text { (2016). }\end{array}$ \\
\hline Total NCC & $\begin{array}{l}\text { Total neural correlate of } \\
\text { consciousness }\end{array}$ & $\begin{array}{l}\text { The totality of neural processes } \\
\text { sufficient for a given conscious } \\
\text { state. }\end{array}$ & $\begin{array}{l}\text { Term introduced in Chalmers } \\
(2000) \text {. The total NCC includes } \\
\text { the core NCC together with all } \\
\text { other neurally enabling } \\
\text { conditions (relegated to the } \\
\text { background of the core } \\
\text { processes). }\end{array}$ \\
\hline
\end{tabular}

Figure 1: Kinds of neural correlates of consciousness (The yellow fields contain the proposed reconceptualization; the remaining concepts were introduced in seminal papers of the NCC debate.) 


\section{3 gNCC and some prominent contemporary theo- ries of perceptual consciousness}

Although the gNCC/NCc distinction does not depend on any particular theory of consciousness, it is not formulated independently of empirical research. On the contrary, some of the most prominent current theories of consciousness permit to distinguish between the content-constituting mechanisms and the gNCC. In the following, we will selectively review five examples of three types of theories of consciousness: those that support, those that probably do not support and those that almost certainly do not support the gNCC notion.

Prinz (2012) explicitly recommends to distinguish between these two mechanisms in his attentional theory of consciousness (Attended intermediate-level representation theory - AIR). The AIR theory's basic claim is that phenomenal consciousness arises if and only if the intermediate-level representations are modulated by attention (Prinz, 2012, p. 89). The intermediate level of representations resides in between the too detailed and pointilistic representations in the early visual areas and the too abstract, categorial representations in the higher visual areas. At the cellular level, the intermediate-level visual representations, the contents of experience, are implemented in the pyramidal neurons within the visual cortex. Combining population and temporal coding, Prinz coins the term "vectorwaves". A vectorwave is a pattern of activity in a population of neurons, a pattern with distinctive temporal dynamics. Vectorwaves implement perceptual contents: red colour is implemented by a particular vectorwave, and so is blue or yellow colour. For colours to become conscious, though, more is needed than just the population's firing in a vectorwave pattern. It is necessary that a synchronous firing of the vectorwave neurons in the gamma range (25 to $70 \mathrm{~Hz}$ oscillations) takes place. Neurally, this synchronization is triggered by interneurons connected to the visual pyramidal cells and modulating their activity by inhibitory mechanisms (Prinz, 2012, p. 135); both pyramidal neurons and the interneurons are localized in the same cortex area. Putting the two distinct neural processes, vectorwaves and gamma synchronization, together, Prinz speaks about "gamma vectorwaves". In our terms, a gamma vectorwave is the NCcc of a particular colour experience, and it is composed of the vectorwave fixing the perceptual content (NCc) and the synchronous gamma oscillation (gNCC) allowing the content to become conscious.

The Global Neuronal Workspace Theory (GNWT) comports with our conceptualization, too. The theory identifies the neural substrate of consciousness itself with activation of the "global workspace" consisting mainly of long-distance axonal connections of large pyramidal neurons in cortical layers II and III (Dehaene \& Changeux, 2011; Mashour et al., 2020; Sergent \& Naccache, 2012). First, the signal travels through visual areas and is further amplified by local feedback loops. Visual contents generated by this processing remain unconscious. Provided the signal is sufficiently strong, it travels onwards to the prefrontal cortex. Longdistance axons of the pyramidal neurons then secure feedback connections from 
the prefrontal cortex to lower areas, especially to associative areas in the posterior parietal cortex and also all the way back to the visual areas. This "ignition" (Dehaene et al., 2003) of the neuronal workspace results in distributed yet synchronized oscillatory activity and makes the visual signals available for other modules connected by the neuronal workspace. Such global availability, arising between 200 and $280 \mathrm{~ms}$ after stimulus onset, marks a transition towards conscious perception. Indeed, according to the GNWT, the global availability of contents simply is what it means for them to be perceived consciously. The sustained activation of the neuronal workspace thus can be taken to constitute the gNCC according to the GNWT.

The distinction between gNCC and NCc does not smoothly apply to all major theories that currently compete to explain conscious perception, though. Lamme's Recurrent Processing Theory (RPT) is a case in point. On a straightforward reading of RPT, visual contents are made conscious when, after the "feedforward sweep" of visual processing, the signal is sent back from higher visual areas to lower ones. The feedforward sweep through the hierarchy of visual areas activates the visual content mechanisms and gives the perceptual state its particular form. During this initial phase of activation, visual features such as orientation, contrast, colour, or direction of motion are extracted and visual objects are categorized. But all this happens unconsciously. It is the feedback from higher to lower areas that turns unconscious vision into conscious seeing. Feedback from higher to lower areas thus can be taken to be the gNCC according to the RPT.

This straightforward reading is supported by Lamme $(2003,2006)$. At one place Lamme openly states that " $[\mathrm{w}] \mathrm{e}$ could even define consciousness as recurrent processing” (Lamme, 2006, p. 499). However, the story in Lamme's more recent article (Lamme, 2020) takes a different turn. Here Lamme attributes complex visual functions to recurrent processing: the function of perceptual organization, mainly of the figure-ground segregation, and of object integration. None of these functions can be implemented by the feedforward sweep itself. Although recurrent processing in this account remains to be tightly associated with consciousness (because none of the complex visual functions can be realized unconsciously), it now has a distinctly visual form. Feedback from higher to lower areas does not constitute a content-nonspecific gNCC, but is just a final stage of visual processing. On such a view, vision has no need for a true gNCC: visual consciousness is the automatic outcome of the latest stages of visual processing. The content mechanisms cannot be separated from recurrent processing because recurrent processing has a visual representational nature, taking care of the visual figure-ground segregation and visual object integration.

RPT in its latest form thus does not support the gNCC idea. The straightforward reading, taking recurrent processing to be the gNCC, would now be rejected by Lamme. Still, let us try to offer an argument in its favour. In a recent intermodality comparison study of neural substrates of conscious perception, Auksztulewicz et al. (2012) compared the neural correlates of visual and tactile percepts. 
Their presumed gNCC was recurrent cortical loops, the same gNCC as in the RPT (on the straightforward reading). Of the three models Auksztulewicz et al. used to interpret their data, feedforward, feedback and recurrent, the recurrent model best fitted the somatosensory detection task data for data segments longer than 140ms. If recurrent loops between somatosensory regions are responsible for making tactile contents conscious, there is reason to suppose that recurrent processing is a domain-neutral mechanism, just as would be expected from a true gNCC. Treating recurrent processing as a content mechanism, as Lamme (2020) does, is inconsistent with such inter-domain evidence.

The Integrated Information Theory of consciousness (IIT; Tononi, 2004; Oizumi et al., 2014; Tononi et al., 2016) is even less compatible with the gNCC/NCc distinction. IIT states that both conscious and unconscious processing lead to the integration of information within the brain. The difference between the two kinds of processing lies in the degree of integration. If the level of information integration during perception is too low to change the contents into conscious contents (clustered in a mental state), perception remains subliminal. A higher degree of information integration leads to conscious perception. IIT further suggests that a system with a purely feedforward architecture cannot generate conscious states (Oizumi et al., 2014, p. 19).

If a feedforward architecture is not enough to turn contents into conscious contents, might not some sort of feedback processing be this additional gNCC mechanism? Although Oizumi et al. (2014) appreciate the value of feedback processing for visual perception, the answer is no. Feedback processing cannot be the IIT version of gNCC because even without actual feedback processing a neuronal informational "complex" can be conscious. From the perspective of the theory, the potential for local interactions among the parts of the complex is more important for consciousness than actual recurrent processing among its parts (Oizumi et al., 2014, p. 20); "if all the neurons in a main complex were inactive (or active at a low baseline rate), they would still generate consciousness as long as they are ready to respond to incoming spikes" (Oizumi et al., 2014, p. 17).

This is one reason why IIT does not support the gNCC idea. The second one is that at least on some versions of IIT, perceptual content mechanisms are inextricably interwoven with consciousness-conferring processes. This is the case in Tsuchiya's account, for example. Tsuchiya (2017, p. 4) comments on the role of global availability of information and of recurrent processing in explanations of conscious perception. These processes, Tsuchiya believes, cannot account for the phenomenological differences between experiences.

Why does vision feel like vision? Within vision, why does color feel different from shape, despite both being generated in the visual cortex? Whatever is critical for consciousness should be specific for the cortico-thalamic system during the awake and the dreaming state and should be differentiable in ways that allow us to understand different modalities and their particular characteristics. (Tsuchiya, 2017, p. 4) 
Indeed, recurrent processing is shared among qualitatively different experiences, and the same holds for the global availability of information (in the GNWT story). But notice that if the distinction between gNCC and NCc is accepted, explaining the phenomenological differences between experiences stops being the job of either the RPT (on the straightforward reading) or of the GNWT. Their job is merely to explicate how various perceptual contents become conscious - to describe the gNCC. The phenomenological questions Tsuchiya raises, although central for the science of consciousness, must be tackled by a radically different type of a theory.

Finally, no application may be given to the gNCC idea in pure sensory processing theories of consciousness such as in Zeki and Bartels' theory of essential nodes (Zeki \& Bartels, 1999). The authors claim that some perceptual processing sites in the visual cortex are at the same time the sites of conscious processing. When the neural signal reaching these "essential nodes" is sufficiently strong, the nodes generate a full-blown content of the particular feature that is processed in the site, such as colour (in the areas V4 and V4 $\alpha$ ) or motion perception (in V5/MT). At the same time, a (micro)consciousness for each of these features is produced in the same node as well. The theory pictures consciousness as disunified at first because all the distinct visual features of a mental state are processed and made conscious independently of each other. All this happens within the $500 \mathrm{~ms}$ after stimulus onset (Zeki, 2003) without our noticing it introspectively. The microconsciousnesses are then bound together to form a unified conscious percept.

Clearly, the unifying mechanism putting the individual contents together cannot be the gNCC. When it starts its work, consciousness, although distributed and not yet unified, is already present. Given that no recurrence, feedback or any other additional mechanism is needed for a piece of perceptual content to become conscious, we see no space for a dissociable gNCC in the essential nodes theory. For a content to become conscious, increased activity within essential node is all that is needed.

\section{Why countenance the gNCC notion?}

As the previous selective review shows, some theories support the gNCC idea, others do not, and still for others it is unclear. It is therefore a matter of dispute whether a theory of consciousness needs to embrace the gNCC notion. The theories employing (or at least permitting) the gNCC/NCc distinction, especially AIR and GNWT, draw on considerable empirical evidence. But so do the rival theories not supporting the distinction, such as the IIT and the essential nodes theory. In this section, we want to contribute to the debate on this subject by summarizing what we see as the two main theoretical advantages of the theories supporting the distinction between the general neural process of perceptual consciousness and content-related neural processing. 


\begin{tabular}{|l|l|l|}
\hline Theory of consciousness & gNCC mechanism & Proponents \\
\hline $\begin{array}{l}\text { Attended intermediate-level } \\
\text { representation (AIR) theory }\end{array}$ & Yes - gamma synchronies & Prinz (2012) \\
\hline $\begin{array}{l}\text { Global neuronal workspace } \\
\text { theory (GNWT) }\end{array}$ & $\begin{array}{l}\text { Yes - sustained synchronous } \\
\text { oscillatory activity in the } \\
\text { neuronal workspace }\end{array}$ & $\begin{array}{l}\text { Dehaene \& Changeux (2011) } \\
\text { Sergent \& Naccache (2012) } \\
\text { Mashour et al. (2020) }\end{array}$ \\
\hline $\begin{array}{l}\text { Recurrent processing theory } \\
\text { (RPT) }\end{array}$ & No (?) & $\begin{array}{l}\text { Lamme (2020) } \\
\text { vs Lamme (2003) and (2006) }\end{array}$ \\
\hline $\begin{array}{l}\text { Information integration theory } \\
\text { (IIT) }\end{array}$ & No (?) & $\begin{array}{l}\text { Tononi (2004) } \\
\text { Oizumi et al. (2014) } \\
\text { Tsuchiya (2017) }\end{array}$ \\
\hline Essential nodes theory & No & Zeki \& Bartels (1999) \\
\hline
\end{tabular}

Figure 2: Selected theories of perceptual consciousness and the gNCC

(i) A strength of a theory supporting the gNCC notion is that it allows a mental state to be fully formed in all its perceptual aspects without becoming conscious. Perceptual processing is necessary but not sufficient for consciousness. In contrast, theories such as the essential nodes theory or the IIT claim that perceptual contents become conscious just by being perceptually constituted in a certain way - by having a sufficiently strong input into the essential node or reaching a sufficiently high level of information integration. In these theories, perceptual processing is both necessary and sufficient for consciousness. A problem for the latter family of theories is that even if the input signals are sufficiently strong, and remain sufficiently stable and distinct, the contents might not become conscious (Cleeremans, 2014; Frith, 2019). Experimental paradigms such as Continuous Flash Suppression (Faivre et al., 2014; Sterzer et al., 2014) or change blindness (Beck et al., 2001; Reddy et al., 2006; Simons \& Rensink, 2005) allow experimenters to keep perceptual processing in place while not letting perceptual contents enter the stream of consciousness. Perceptual contents are still being constituted and poised for becoming conscious; but they do not become conscious.

To take an example, Jiang et al. (2009) used a dichoptic masking paradigm to measure temporal dynamics of facial information processing. In one of the experiments, the subjects were perceiving a face with neutral expression in two conditions, visible and invisible. In the invisible condition, processing of face-related attributes was reduced (as documented by a decrease in amplitudes of the ERP components). Still, in both conditions, the experimenters recorded a significant similarity of positive (P1) and negative (N1) components of the ERP waveform. (The compared waveforms started around $220 \mathrm{~ms}$ after stimulus onset in both visible 
and invisible condition). The authors consider this to be evidence for unconscious cortical processing of faces in superior temporal sites (Jiang et al., 2009, p. 1176). ${ }^{3}$

From the perspective of this paper, the most interesting possibility of this sort is when the perceptual contents mechanisms are functioning well but as a result of the gNCC itself being disrupted, the contents do not reach consciousness. For instance, artificial disruption of feedback loops from MT+/V5 to V1 by transcranial magnetic stimulation causes loss of visual consciousness of motion (Pascual-Leone \& Walsh, 2001). The gNCC idea provides a simple explanation. Feedback from higher to lower areas is the gNCC. Disruption of feedback loops from MT+/V5 to V1 thus amounts to the disruption of gNCC itself. Resulting loss of conscious perception of motion is inevitable even if the processing at perceptual site (MT+/V5) remains intact. The feedback signals are not participating in perceptual processing but act as a consciousness conferring mechanism.

Such disruption of the gNCC should be possible - and empirically testable - in other theories of consciousness supporting the gNCC/NCc distinction. Recall that gamma synchronisation is the gNCC according to AIR theory. Disrupting it should stop the stream of colour consciousness even if visual content correlates - the vectorwaves - continue to process incoming signals. In fact, Prinz (2015, p. 384) considers this possibility. Vectorwaves can be activated without the content they carry becoming conscious. Visual contents can be formed unconsciously but fail to reach awareness if gamma synchronization fails. When a particular neuronal population fires out of synchrony, we may perceive blue but only unconsciously. Similarly, according to the GNWT, the long-range axonal connections between prefrontal and parieto-temporal cortex secure wide distribution of sensory contents. Disrupting these long-range connections (i.e., disrupting the GNWT's gNCC) should disrupt conscious perception. Meanwhile, the sensory areas processing visual and other stimuli can still be working; processing in the sensory areas can go deep and significantly influence behaviour even when the signals ultimately do not reach consciousness (Dehaene et al., 1998; Dehaene, 2014; Sergent et al., 2005). Again, sensory contents could be completely processed at the perceptual level while not entering into conscious stream. ${ }^{4}$

(ii) Being strict about keeping the processing of perceptual contents apart from consciousness-related processing in one's theory is also an important pre-

\footnotetext{
${ }^{3}$ It has to be noted that the extent of unconscious processing under various experimental paradigms is the subject of ongoing controversy. In particular, while there is a general agreement that lower level visual features such as stimulus orientation can be perceived unconsciously under Continuous Flash Suppression, unconscious perception of higher-level features, such as face identity or facial expression, was not demonstrated beyond reasonable doubt. For the most comprehensive recent review of Continuous Flash Suppression studies, and the controversies surrounding the results, see Pournaghdali \& Schwartz (2020).

${ }^{4}$ The signals in sensory areas during unconscious processing are weaker then during conscious processing. This does not contradict our hypothesis. The weakening of the signals in unconscious conditions could be explained by the absence of the gNCC activation. For more details, (see Marvan \& Polák, 2017).
} 
requisite for building a truly general, pan-modality theory of conscious perception. Although most of the theories of consciousness are applied to visual data, this limitation must be overcome. It would be desirable to have a systematic and unifying theory of what makes visual, auditory, gustatory and other forms of contents conscious. The gNCC idea is a perfect match for this ambitious project, being completely modality-neutral.

Of the theories discussed in the previous section 3, the GNWT is already formulated in a domain-general way. The neuronal workspace globally distributes visual, auditory, tactile or olfactory contents (Dehaene, 2014, pp. 13, 119-120, 158, 217, and 221). There is no specialized gNCC for vision, distinct from gNCC for audition etc. ${ }^{5}$ Unfortunately, there are almost no other attempts to identify a shared gNCC in different sensory modalities. Apart from the promising visual-tactile comparison study mentioned in section 3 (Auksztulewicz et al., 2012), initial comparisons of visual and auditory correlates of conscious perception were performed by Snyder et al. (2015). According to them, auditory Awareness-Related Negativity, a hypothesized marker of conscious hearing, is the auditory analogue of the Visual Awareness Negativity, a presumed signature of arising visual consciousness. AwarenessRelated Negativity typically peaks around $100 \mathrm{~ms}$ after the stimulus onset, Visual Awareness Negativity around 50-100ms later. Both negativities consistently show up in EEG and MEG studies of conscious perception in both domains, displaying robust invariance under different experimental paradigms and changing task demands. This is not just an interesting result in its own right, but indirectly points to the role of recurrent processing, which is often taken to be the correlate of Visual Awareness Negativity (see Förster et al., 2020). Recurrent processing theories of consciousness (such as the RPT in the straightforward reading) thus gain further evidential support.

More comparative inter-modality research will show whether the results generalize and vindicate the idea of a unique gNCC mechanism. The guiding principle of any such research must be to carefully disentangle the presumed gNCC process from an almost indefinite variety of content endowing mechanisms. In the words of Snyder and colleagues:

Paradigms designed to isolate neural correlates of the contents of consciousness are likely to find different neuroanatomical sources for auditory versus visual NCCs. By contrast, studies that are able to isolate neural mechanisms that bring these contents into awareness, which may be a distinct process, could reveal overlapping neural sources between auditory and visual modalities. (Snyder et al., 2015, p. 296)

The only difference between this view and our proposal is that we do not insist on literally the same neurophysiological resources of consciousness-conferring mech-

\footnotetext{
${ }^{5}$ In fact, GNWT has even broader ambitions in some of its formulations. Influential GNW theorists claim that the workspace idea can be extended to "felt emotions, visual imagery, working memory, and executive control" (Baars et al., 2013).
} 
anism being deployed in different modalities. This would indeed be the case of GNWT where different ignitions of the global workspace may literally use overlapping neuronal coalitions to broadcast contents from different sensory modalities. But this is not required for a gNCC. What matters is merely that the same type of neural mechanism is deployed across perceptual episodes in different modalities. The gNCC mechanism for different modalities may be localized non-overlapping areas. In fact, this is the picture behind AIR. The theory would probably locate gamma synchronies in different dedicated sensory cortices for different modalities - in the visual cortex for conscious vision, in the auditory cortex for conscious audition etc. ${ }^{6}$

\section{Concluding remarks}

While the search for neural correlates of conscious perception is an empirical enterprise, it benefits from theoretical-conceptual and methodological reflections. The hypothesis put forward in this article is that the standard notion of perceptual NCC conflates the processing of perceptual contents with consciousness conferring mechanisms. Whereas the content mechanisms (NCc) secure all the richness of experience, gNCC is a content-free, domain-neutral mechanism. The resulting neural correlate of a specific type of experienced content (NCcc) arises in the dynamic interaction between these two distinct types of processes.

Although we propose a revision of the content-based NCC concept, the proposal is motivated by theoretical and empirical considerations and does not have the form of a conceptual truth. Consequently, our goal in this paper was modest: to open the discussion on the possibility of the gNCC/NCc distinction and to marshal some of the initial arguments in its favour. In the remainder, let us point out two advantages of endorsing it.

First, the theories supporting the gNCC/NCc distinction are amenable to direct testing in terms of the hypothesized gNCC. If during testing the presumed gNCC is either detected in conditions of subliminal perception, or if conscious perception occurs in the absence of the gNCC, the tested theory is deficient. ${ }^{7}$ On the other hand, evidence against the gNCC according to a particular theory does not disqualify the gNCC idea as such. The gNCC might be implemented elsewhere in the brain, or in a different way altogether.

Secondly, the gNCC/NCc distinction helps to appreciate that tracing the content mechanisms and elucidating how they work may be a different project from

\footnotetext{
${ }^{6}$ We say "would probably locate” because Prinz (2012) does not extend his gamma synchrony theory to non-visual modalities.

${ }^{7}$ For the first possibility see, e.g., the GNWT-contradicting evidence in Silverstein et al. (2015) that P3b, an ERP index of workspace ignition (Dehaene et al., 2003), may be present under rigorously subliminal conditions. For the second possibility, see the AIR-contradicting evidence that increase in local gamma synchronisation might not be sufficient for conscious face perception in Aru et al. (2012).
} 
the effort to isolate the general mechanism by which the contents are made conscious. In our estimate, the first of the two research projects will be much more difficult to carry through. The questions falling to the content side belong to the most vexing issues of the science of consciousness. Why are perceptual contents constituted in one way and not another? Why does hearing the sound of trumpet feel so different from smelling fresh strawberries, given the similarity of the underlying neural processing in both cases? Where are the contents located? Do only striate and extrastriate areas have the neural resources to express the visual contents down to their very fine details, or may these contents be expressed elsewhere in the brain? Are the consciously experienced contents the very same as those formed unconsciously? Or is the activity in sensory areas a necessary prerequisite but not a proper neural substrate of conscious perception? In comparison with these riddles, the search for the gNCC may turn out to be a more manageable enterprise. As the proposals discussed in section 3 indicate, the gNCC could be a mechanism having the role of boosting or distributing the sensory signals.

\section{Acknowledgments}

We would like to thank the anonymous reviewers for thoughtful comments and to Sascha Benjamin Fink, Wanja Wiese and Jennifer Windt for helpful suggestions and outstanding editorial work. This article is the outcome of the Czech Science Agency (GAČR) project n. 20-14445S "Dual Models of Phenomenal Consciousness".

\section{References}

Aru, J., Axmacher, N., Do Lam, A. T. A., Fell, J., Elger, C. E., Singer, W., \& Melloni, L. (2012). Local category-specific gamma band responses in the visual cortex do not reflect conscious perception. The fournal of Neuroscience, 32(43), 1490914914. https://doi.org/10.1523/JNEUROSCI.2051-12.2012

Aru, J., Bachmann, T., Singer, W., \& Melloni, L. (2012). Distilling the neural correlates of consciousness. Neuroscience and Biobehavioral Reviews, 36(2), 737-746. https://doi.org/10.1016/j.neubiorev.2011.12.003

Auksztulewicz, R., Spitzer, B., \& Blankenburg, F. (2012). Recurrent neural processing and somatosensory awareness. The fournal of Neuroscience, 32(3), 799-805. https://doi.org/10.1523/JNEUROSCI.3974-11.2012

Baars, B. J., Franklin, S., \& Ramsoy, T. Z. (2013). Global workspace dynamics: Cortical "binding and propagation” enables conscious contents. Frontiers in Psychology, 4, 200. https://doi.org/10.3389/fpsyg.2013.00200

Bayne, T., \& Hohwy, J. (2013). Consciousness: Theoretical approaches. In A. E. Cavanna, A. Nani, H. Blumenfeld, \& S. Laureys (Eds.), Neuroimaging of consciousness (pp. 23-35). Springer.

Bayne, T., Hohwy, J., \& Owen, A. M. (2016). Are there levels of consciousness? Trends in Cognitive Sciences, 20(6), 405-413. https://doi.org/10.1016/j.tics.2016.03.009

Beck, D. M., Rees, G., Frith, C. D., \& Lavie, N. (2001). Neural correlates of change detection and change blindness. Nature Neuroscience, 4(6), 645-650. https://doi.org/10.1038/88477

Block, N. (2005). Two neural correlates of consciousness. Trends in Cognitive Sciences, 9(2), 46-52. https://doi.org/10.1016/ j.tics.2004.12.006

Boly, M., Massimini, M., Tsuchiya, N., Postle, B. R., Koch, C., \& Tononi, G. (2017). Are the neural correlates of consciousness in the front or in the back of the cerebral Cortex? Clinical and neuroimaging evidence. The fournal of Neuroscience, 37(40), 9603-9613. https://doi.org/10.1523/JNEUROSCI.3218-16.2017

Chalmers, D. J. (2000). What is a neural correlate of consciousness? In T. Metzinger (Ed.), Neural correlates of consciousness (pp. 17-39). MIT Press.

Cleeremans, A. (2014). Prediction as a computational correlate of consciousness. International fournal of Computing Anticipatory Systems, 29, 3-13. https://axc.ulb.be/uploads/2016/01/14-casys11.pdf

Crick, F. H. C. (1994). The astonishing hypothesis: The scientific search for the soul. Charles Scribner's Sons.

Marvan, T., \& Polák, M. (2020). Generality and content-specificity in the study of the neural correlates of perceptual consciousness. Philosophy and the Mind Sciences, 1(II), 5. 
Dehaene, S. (2014). Consciousness and the brain: Deciphering how the brain codes our thoughts. Penguin Books.

Dehaene, S., \& Changeux, J.-P. (2011). Experimental and theoretical approaches to conscious processing. Neuron, 70(2), 200-227. https://doi.org/10.1016/j.neuron.2011.03.018

Dehaene, S., Naccache, L., Le Clec'H, G., Koechlin, E., Mueller, M., Dehaene-Lambertz, G., Moortele, P. F. van de, \& Le Bihan, D. (1998). Imaging unconscious semantic priming. Nature, 395(6702), 597-600. https://doi.org/10.1038/26967

Dehaene, S., Sergent, C., \& Changeux, J.-P. (2003). A neuronal network model linking subjective reports and objective physiological data during conscious perception. Proceedings of the National Academy of Sciences, 100(14), 8520-8525. https://doi.org/10.1073/pnas.1332574100

Faivre, N., Berthet, V., \& Kouider, S. (2014). Sustained invisibility through crowding and continuous flash suppression: A comparative review. Frontiers in Psychology, 5, 475. https://doi.org/10.3389/fpsyg.2014.00475

Fink, S. B. (2016). A deeper look at the neural "correlate of consciousness". Frontiers in Psychology, 7, 1044. https://doi.org/ 10.3389/fpsyg.2016.01044

Förster, J., Koivisto, M., \& Revonsuo, A. (2020). ERP and MEG correlates of visual consciousness: The second decade. Consciousness and Cognition, 80, 102917. https://doi.org/10.1016/j.concog.2020.102917

Frith, C. D. (2019). The neural basis of consciousness. Psychological Medicine, 1-13. https://doi.org/10.1017/S00332917190 02204

Haynes, J.-D. (2009). Decoding visual consciousness from human brain signals. Trends in Cognitive Sciences, 13(5), 194-202. https://doi.org/10.1016/j.tics.2009.02.004

Hohwy, J. (2009). The neural correlates of consciousness: New experimental approaches needed? Consciousness and Cognition, 18(2), 428-438. https://doi.org/10.1016/j.concog.2009.02.006

Jiang, Y., Shannon, R. W., Vizueta, N., Bernat, E. M., Patrick, C. J., \& He, S. (2009). Dynamics of processing invisible faces in the brain: Automatic neural encoding of facial expression information. NeuroImage, 44(3), 1171-1177. https: //doi.org/10.1016/j.neuroimage.2008.09.038

Keaton, D. (2015). Constitution, realization and identity. In S. Miller (Ed.), The constitution of phenomenal consciousness: Toward a science and theory (pp. 372-399). John Benjamins.

Koch, C. (2004). The quest for consciousness: A neurobiological approach. Roberts \& Company.

Koch, C., Massimini, M., Boly, M., \& Tononi, G. (2016). Neural correlates of consciousness: Progress and problems. Nature Reviews. Neuroscience, 17(5), 307-321. https://doi.org/10.1038/nrn.2016.22

Lamme, V. A. (2003). Why visual attention and awareness are different. Trends in Cognitive Sciences, 7(1), 12-18. https: //doi.org/10.1016/s1364-6613(02)00013-x

Lamme, V. A. F. (2006). Towards a true neural stance on consciousness. Trends in Cognitive Sciences, 10(11), 494-501. https://doi.org/10.1016/j.tics.2006.09.001

Lamme, V. A. F. (2020). Visual Functions Generating Conscious Seeing. Frontiers in Psychology, 11, 83. https://doi.org/10.3 389/fpsyg.2020.00083

Marvan, T., \& Polák, M. (2017). Unitary and dual models of phenomenal consciousness. Consciousness and Cognition, 56, 1-12. https://doi.org/10.1016/j.concog.2017.09.006

Mashour, G. A., Roelfsema, P., Changeux, J.-P., \& Dehaene, S. (2020). Conscious processing and the global neuronal workspace hypothesis. Neuron, 105(5), 776-798. https://doi.org/10.1016/j.neuron.2020.01.026

McKilliam, A. (2020). What is a global state of consciousness? Philosophy and the Mind Sciences, 1(II), 1-17. https://doi.or g/https://doi.org/10.33735/phimisci.2020.II.58

Michel, M., \& Morales, J. (2020). Minority reports: Consciousness and the prefrontal cortex. Mind and Language, 35(4), 493-513. https://doi.org/10.1111/mila.12264

Miller, S. (2015). The correlation/constitution distinction problem: Foundations, limits and explanation in consciousness science. In S. Miller (Ed.), The constitution of phenomenal consciousness: Toward a science and theory (pp. 104-154). John Benjamins.

Oizumi, M., Albantakis, L., \& Tononi, G. (2014). From the phenomenology to the mechanisms of consciousness: Integrated Information Theory 3.0. PLoS Computational Biology, 10(5), e1003588. https://doi.org/10.1371/journal.pcbi.1003588

Pascual-Leone, A., \& Walsh, V. (2001). Fast backprojections from the motion to the primary visual area necessary for visual awareness. Science, 292(5516), 510-512. https://doi.org/10.1126/science.1057099

Pournaghdali, A., \& Schwartz, B. L. (2020). Continuous flash suppression: Known and unknowns. Psychonomic Bulletin \& Review, 27(6), 1071-1103. https://doi.org/10.3758/s13423-020-01771-2

Prinz, J. (2012). The conscious brain. Oxford University Press.

Prinz, J. J. (2015). Unconscious perception. In M. Matthen (Ed.), The Oxford handbook of philosophy of perception. (pp. 371-389). Oxford University Press.

Marvan, T., \& Polák, M. (2020). Generality and content-specificity in the study of the neural correlates of perceptual consciousness. Philosophy and the Mind Sciences, 1(II), 5.

https://doi.org/10.33735/phimisci.2020.II.61

(c) (1) (c) The author(s). https://philosophymindscience.org ISSN: 2699-0369 
Reddy, L., Quiroga, R. Q., Wilken, P., Koch, C., \& Fried, I. (2006). A single-neuron correlate of change detection and change blindness in the human medial temporal lobe. Current Biology, 16(20), 2066-2072. https://doi.org/10.1016/j.cub.2006 .08 .064

Rees, G., Kreiman, G., \& Koch, C. (2002). Neural correlates of consciousness in humans. Nature Reviews. Neuroscience, 3(4), 261-270. https://doi.org/10.1038/nrn783

Sandberg, K., Andersen, L. M., \& Overgaard, M. (2014). Using multivariate decoding to go beyond contrastive analyses in consciousness research. Frontiers in Psychology, 5, 1250. https://doi.org/10.3389/fpsyg.2014.01250

Sergent, C., Baillet, S., \& Dehaene, S. (2005). Timing of the brain events underlying access to consciousness during the attentional blink. Nature Neuroscience, 8(10), 1391-1400. https://doi.org/10.1038/nn1549

Sergent, C., \& Naccache, L. (2012). Imaging neural signatures of consciousness: "What", "when", "where" and "how" does it work? Archives Italiennes de Biologie, 150(2-3), 91-106. https://doi.org/10.4449/aib.v150i2.1270

Silverstein, B. H., Snodgrass, M., Shevrin, H., \& Kushwaha, R. (2015). P3b, consciousness, and complex unconscious processing. Cortex, 73, 216-227. https://doi.org/10.1016/j.cortex.2015.09.004

Simons, D. J., \& Rensink, R. A. (2005). Change blindness: Past, present, and future. Trends in Cognitive Sciences, 9(1), 16-20. https://doi.org/10.1016/j.tics.2004.11.006

Snyder, J. S., Yerkes, B. D., \& Pitts, M. A. (2015). Testing domain-general theories of perceptual awareness with auditory brain responses. Trends in Cognitive Sciences, 19(6), 295-297. https://doi.org/10.1016/j.tics.2015.04.002

Sterzer, P., Stein, T., Ludwig, K., Rothkirch, M., \& Hesselmann, G. (2014). Neural processing of visual information under interocular suppression: A critical review. Frontiers in Psychology, 5, 453. https://doi.org/10.3389/fpsyg.2014.00453

Tononi, G. (2004). An information integration theory of consciousness. BMC Neuroscience, 5, 42. https://doi.org/10.1186/14 71-2202-5-42

Tononi, G., Boly, M., Massimini, M., \& Koch, C. (2016). Integrated information theory: From consciousness to its physical substrate. Nature Reviews Neuroscience, 17(7), 450-461. https://doi.org/10.1038/nrn.2016.44

Tsuchiya, N. (2017). "What is it like to be a bat?"-a pathway to the answer from the integrated information theory. Philosophy Compass, 12(3), e12407. https://doi.org/10.1111/phc3.12407

Tsuchiya, N., Wilke, M., Frässle, S., \& Lamme, V. A. F. (2015). No-report paradigms: Extracting the true neural correlates of consciousness. Trends in Cognitive Sciences, 19(12), 757-770. https://doi.org/10.1016/j.tics.2015.10.002

Zeki, S. (2003). The disunity of consciousness. Trends in Cognitive Sciences, 7(5), 214-218. https://doi.org/10.1016/S13646613(03)00081-0

Zeki, S., \& Bartels, A. (1999). Toward a theory of visual consciousness. Consciousness and Cognition, 8(2), 225-259. https: //doi.org/10.1006/ccog.1999.0390

\section{Open Access}

This article is distributed under the terms of the Creative Commons Attribution 4.0 International License (https://creativecommons.org/licenses/by/4.0/), which permits unrestricted use, distribution, and reproduction in any medium, as long as you give appropriate credit to the original author(s) and the source, provide a link to the Creative Commons license, and indicate if changes were made.

Marvan, T., \& Polák, M. (2020). Generality and content-specificity in the study of the neural correlates of perceptual consciousness. Philosophy and the Mind Sciences, 1(II), 5. 